\title{
Perfect Equilibria in a Negotiation Model With Different Time Preferences \\ by
}

Harold Houba and Quan Wen

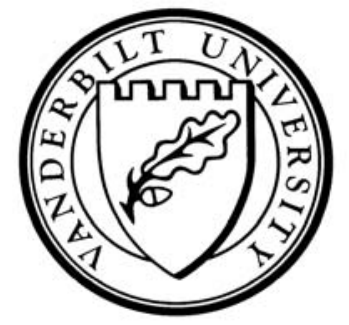

Working Paper No. 07-W06

April 2007

\section{DEPARTMENT OF ECONOMICS \\ VANDERBILT UNIVERSITY \\ NASHVILLE, TN 37235}

www.vanderbilt.edu/econ 


\title{
Perfect Equilibria in a Negotiation Model with Different Time Preferences*
}

\author{
Harold Houba ${ }^{\dagger}$ \\ Vrije Universiteit \\ and Tinbergen Institute \\ Quan Wen $\ddagger$ \\ Vanderbilt University \\ First version: March 2006 \\ Revised version: April 2007
}

\begin{abstract}
The players behave quite differently in the negotiation model under different time preferences than under common time preferences. Conventional analysis in this literature relies on the key presumption that all continuation payoffs are bounded from above by the bargaining frontier resulted from stationary contracts. When players have different time preferences, however, intertemporal trade may lead to continuation payoffs above the bargaining frontier. In this paper, we provide a thorough study of this problem when players have different time preferences. Our results tie up all the previous findings, and also clarify the confusion that arose in the past.
\end{abstract}

JEL Classification: C72 Noncooperative Games, C73 Stochastic and Dynamic Games, C78 Bargaining Theory

Keywords: Bargaining, negotiation, time preference, endogenous threats

\footnotetext{
${ }^{*}$ We would like to thank three anonymous referees and a co-editor for many helpful comments and suggestions. This project is partially supported by Netherlands Organization for Scientific Research (NWO), grant B45-271. Quan Wen is grateful to Gerard van der Laan, the Tinbergen Institute and the Vrije Universiteit for their hospitality and generosity.

${ }^{\dagger}$ Department of Econometrics, Vrije Universiteit, De Boelelaan 1105, 1081 HV Amsterdam, Netherlands. Email: hhouba@feweb.vu.nl.

${ }^{\ddagger}$ Department of Economics, Vanderbilt University, VU Station B \#351819, 2301 Vanderbilt Place Nashville, TN 37235-1819, U.S.A. Email: quan.wen@vanderbilt.edu.
} 


\section{Introduction}

Endogenous threats are an essential constituent of bargaining problems, as emphasized in Nash (1953) at the dawn of modern bargaining theory. The bargaining literature in the 1990s successfully incorporates endogenous threats into the alternating-offer bargaining model of Rubinstein (1982). The early contributions in this area, such as Fernandez and Glazer (1991), Haller (1991) and Haller and Holden (1990), study the selection of industrial action by a union during its contract negotiations with a firm. In contrast to Rubinstein (1982), after a proposal is rejected, the union needs to decide what course of industrial action to take before the next bargaining round. ${ }^{1}$ Later contributions, such as Busch and Wen (1995, 2001), Houba (1997), and Slantchev (2003), allow for more general forms of endogenous threats, modeled as a normal-form game, called the disagreement game, to be played between offers and counteroffers. ${ }^{2}$ All these references are restricted to stationary contracts, ${ }^{3}$ and except Fernandez and Glazer (1991), Muthoo (1999) and Slantchev (2003), to the case of common time preferences.

Despite our well-understanding of the negotiation model with common time preferences, it is not trivial to generalize the existing analysis and technique to the case with different time preferences. In general, this class of games admits multiple equilibria and the set of the equilibrium payoffs is fully characterized by so-called extreme equilibria that yield the lowest and highest equilibrium payoffs to each player. Bolt (1995) demonstrates that the strategy profile supporting the firm's worst equilibrium provided by Fernandez and Glazer (1991) fails to be an equilibrium when the firm is less patient than the union. He then provides a noconcession strategy to the firm and shows that it can be sustained in equilibrium. Recently, one of the claims by Slantchev (2003) suggests that the firm's no-concession strategy always supports the firm's worst equilibrium when the firm is less patient than the union. This

\footnotetext{
${ }^{1}$ Fernandez and Glazer (1991), Haller (1991), and Haller and Holden (1990) consider two industrial actions. Houba and Bolt (2000) consider the strategic substitutability among several forms of industrial actions by the union.

${ }^{2}$ This negotiation model is surveyed in Muthoo (1999) and Houba and Bolt (2002).

${ }^{3}$ Houba (1997) considers nonstationary contracts under common time preferences.
} 
implication, however, contradicts another finding reported in Bolt (1993) that an alwaysstrike strategy sometimes yields an even lower payoff to the firm. Instead of invoking the technique of Shaked and Sutton (1984) to derive extreme equilibrium payoffs in this model, Bolt (1995) and Slantchev (2003) simply verify whether a given strategy profile constitutes an equilibrium. Muthoo (1999) also notices the necessity to apply the technique of Shaked and Sutton (1984) in studying the negotiation model under different time preferences.

We treat these findings seriously, not only to settle the open issue of extreme equilibria, but more importantly, to reexamine the method used in previous studies. For more than twenty years, the backward induction technique of Shaked and Sutton (1984) has proven to be a very powerful and effective tool in studying bargaining problems. Applying this technique relies on the presumption that all continuation payoffs are bounded by the bargaining frontier, which holds in most bargaining situations. Consequently, players always reach an immediate agreement in any extreme equilibrium, such as in the original study of Shaked and Sutton (1984). By incorporating the possibility of making unacceptable proposals, Fudenberg and Tirole (1991) show that it is without loss of generality that only acceptable proposals count in applying this technique to the model of Rubinstein (1982).

In the negotiation model with common time preferences, all continuation payoffs are bounded by the bargaining frontier resulting from stationary contracts. Houba and Wen (2006) show that stationary contracts are Pareto efficient if and only if players have common time preferences. However, this is definitely not the case when the players have different time preferences. It has been realized in other dynamic problems that Pareto improvement is possible through intertemporal trade among agents with different time preferences, see e.g., Ramsey (1928), Bewley (1972) and, more recently, Lehrer and Pauzner (1999). In the context of repeated games, Lehrer and Pauzner (1999) demonstrate that many feasible payoffs are outside the conventionally defined set of 'feasible payoffs'. What matters is that, under different time preferences, an infinite sequence of two payoff vectors does not lead to a convex combination of these two vectors. Unlike repeated games, outcome paths in bargaining models are less flexible. Since any agreement on a stationary contract, by 
default, ceases any future payoff variation, an immediate agreement may not be Pareto efficient when players have different time preferences. This is not a serious issue in Rubinstein (1982), because all possible outcome paths are dominated by some immediate agreement. However, in the negotiation model it is possible for both players to benefit from playing some disagreement outcomes for some periods prior to an agreement that rewards the more-patient player. Those benefits can be so dramatic that the resulting continuation payoff vector is above the bargaining frontier. This is exactly what has been overlooked and the root of the problems reported in the current literature.

We do not make any presumption on continuation payoffs. When there are continuation payoffs above the bargaining frontier, players may not always reach an immediate agreement in extreme equilibria. We modify the technique of Shaked and Sutton (1984) with the possibility of unacceptable proposals and obtain a set of necessary and sufficient conditions for the extreme equilibrium payoffs. The Pareto frontier of continuation payoffs plays an essential role in solving these extreme payoffs. In order to achieve this Pareto frontier, it may be necessary to disagree for many periods. In constructing the Pareto frontier from extreme equilibrium payoffs, we borrow and adapt the technique of Lehrer and Pauzner (1999) for the negotiation model. The interdependency between the Pareto frontier and extreme payoffs significantly alters the way we analyze the negotiation model. Although all extreme payoffs are also interdependent, for sufficiently patient players, the worst equilibrium payoff of the less-patient player can be solved without knowing the other extreme equilibrium payoffs. The less-patient player's equilibrium payoffs are bounded from below by the least fixed point of a well-defined minimax problem. We provide an equilibrium that supports such a least fixed point as the less-patient player's worst equilibrium payoff. Furthermore, we provide a simple upper bound for the least patient player's worst equilibrium payoff that is no more than those in Bolt (1995) and Muthoo (1999). To summarize, we develope a general technique to characterize the least patient player's worst equilibrium, from which all other extreme equilibria can be recovered.

In order to demonstrate the technique developed here and its resolution in Fernandez and 
Glazer (1991) and Slantchev (2003), we consider common interest disagreement games where there is a Pareto dominant disagreement outcome. ${ }^{4}$ We find that there are complications only in the worst equilibrium to the less-patient player. The worst equilibrium to the morepatient player closely resembles the worst equilibrium known for common time preferences. This finding validates the firm's worst equilibrium in Fernandez and Glazer (1991) only in case the firm is more patient than the union.

This paper is organized as follows. In Section 2, we present the negotiation model, summarize some existing results, and discuss why some continuation payoffs can be above the bargaining frontier. The analysis is partitioned into three subsections. In Section 3.1, we derive a set of necessary and sufficient conditions for extreme equilibrium payoffs. In order to solve the extreme equilibrium payoffs, we need to know the Pareto frontier of continuation payoffs, which is studied in Section 3.2. In Section 3.3, we derive the worst equilibrium to the proposing player. In Section 4 we focus on the model with common interest disagreement games and show that complications arise only in the worst equilibrium to the less-patient player. We present an example to illustrate our findings. Section 5 offers some concluding remarks.

\section{The Model and Pareto Efficiency}

Consider the negotiation model in which two players, named 1 and 2, negotiate how to split an infinite stream of surpluses of certain value, all normalized to be 1 per period. In any period before reaching an agreement, one player makes a proposal on how to split the value in all future periods and the other player either accepts or rejects the proposal. If the proposal is accepted, then it will be implemented immediately, which ceases any future strategic interaction between the players. If the proposal is rejected, then the players will play a disagreement game once before the negotiations proceed to the following period.

More specifically, the model consists of infinitely many periods where player 1 proposes

\footnotetext{
${ }^{4}$ Common interest games are studied in other dynamic settings, see, e.g., Farrel and Saloner (1985) and Takahashi (2005).
} 
in all odd periods and player 2 proposes in all even periods. A proposal is simply a feasible contract on how to share the future values. As commonly considered in the literature, a proposal is a stationary contract, denoted as

$$
x=\left(x_{1}, x_{2}\right) \in \Delta=\left\{\left(x_{1}, x_{2}\right) \in \mathbb{R}_{+}^{2}: x_{1}+x_{2}=1\right\}
$$

from which player $i$ 's payoff is $x_{i}$ in every period after both players agree on $x \in \Delta$ (the unit simplex), which we refer to as the bargaining frontier. The disagreement game is given in normal form:

$$
G=\left\{A_{1}, A_{2}, d_{1}(\cdot), d_{2}(\cdot)\right\},
$$

where $A_{i}$ is the set of player $i$ 's disagreement actions that is assumed to be non-empty and compact, and $d_{i}(\cdot): A \rightarrow \mathbb{R}$ is player $i$ 's disagreement payoff function that is assumed to be continuous, where $A=A_{1} \times A_{2}$ is the set of disagreement outcomes. We also assume $d_{1}(a)+$ $d_{2}(a) \leq 1$ so that every disagreement outcome is weakly dominated by some agreement. To ease exposition, we denote player $i$ 's highest disagreement payoff when he deviates from $a \in A$ unilaterally by

$$
g_{i}(a)=\max _{a_{i}^{\prime} \in A_{i}} d_{i}\left(a_{i}^{\prime}, a_{j}\right)
$$

We assume that the disagreement game $G$ has at least one Nash equilibrium $a^{N} \in A$ meaning $g_{i}\left(a^{N}\right)=d_{i}\left(a^{N}\right)$ for $i=1$ and 2. Without loss of generality, every player's minimax value in $G$ is normalized to be zero;

$$
\min _{a_{j} \in A_{j}} \max _{a_{i} \in A_{i}} d_{i}(a)=\min _{a \in A} g_{i}(a)=0 .
$$

Let $d(A)$ denote the convex hull of $\{d(a): a \in A\}$, the set of feasible disagreement payoffs.

A generic outcome path in the negotiation model, denoted by $\pi=\left(a^{1}, a^{2}, \cdots, a^{T}, x\right)$ for $T \geq 0$, consists of all disagreement outcomes $\left(a^{t} \in A\right.$ in period $t$ for $\left.t \leq T\right)$ before the agreement $x \in \Delta$ is reached in period $T+1$. Such an outcome path specifies an immediate agreement with $T=0$, and a perpetual disagreement outcome path with $T=\infty$. From such 
an outcome path $\pi$, player $i$ 's intertemporal time preference is represented by his average discounted payoffs from the disagreement game before the agreement and the agreement itself afterward:

$$
v(\pi)=\left(1-\delta_{i}\right) \sum_{t=1}^{T} \delta_{i}^{t-1} d_{i}\left(a^{t}\right)+\delta_{i}^{T} x_{i},
$$

where $\delta_{i} \in(0,1)$ represents player $i$ 's discount factor per period.

The negotiation model described so-far is a well-defined noncooperative game of complete information. A history is a complete description of how the game has been played up to a period. A player's strategy specifies one appropriate action for every finite history. For technical convenience, we allow for public correlated strategies where players can coordinate their continuation strategies based on public coordination devices. Every strategy profile induces a unique distribution on outcome paths and players evaluate their strategies based on their discounted payoffs from the induced outcome paths. The equilibrium concept applied throughout this paper is subgame perfect equilibrium (SPE).

Next, we summarize some existing results for this model from previous studies in the form of two propositions for later reference. We state these results without proof and in terms of player $i \in\{1,2\}$, while refer to his opponent as player $j \neq i$.

Proposition 1 In the negotiation model with different time preferences, for all $\left(\delta_{i}, \delta_{j}\right) \in$ $(0,1)^{2}$, there is a stationary SPE where player $i$ receives

$$
\begin{array}{ll}
d_{i}\left(a^{N}\right)+\frac{1-\delta_{j}}{1-\delta_{i} \delta_{j}}\left[1-d_{i}\left(a^{N}\right)-d_{j}\left(a^{N}\right)\right] & \text { when player } i \text { proposes and } \\
d_{i}\left(a^{N}\right)+\frac{\delta_{i}\left(1-\delta_{j}\right)}{1-\delta_{i} \delta_{j}}\left[1-d_{i}\left(a^{N}\right)-d_{j}\left(a^{N}\right)\right] & \text { when player } j \text { proposes, }
\end{array}
$$

where $a^{N} \in A$ is a Nash equilibrium of $G$.

Proposition 2 In the negotiation model with common time preferences, i.e., $\delta_{i}=\delta_{j}=\delta$, for sufficiently large $\delta \in(0,1)$, player $i$ 's lowest SPE payoff is

$$
\begin{array}{ll}
\frac{1}{1+\delta}\left[1-\max _{a \in A}\left[d_{i}(a)+d_{j}(a)-g_{i}(a)\right]\right] & \text { when player } i \text { proposes and } \\
\frac{\delta}{1+\delta}\left[1-\max _{a \in A}\left[d_{i}(a)+d_{j}(a)-g_{i}(a)\right]\right] & \text { when player } j \text { proposes. }
\end{array}
$$


Propositions 1 and 2 assert that the model generally has multiple SPE payoffs when the players are sufficiently patient. What is less clear is a full characterization of the set of SPE payoffs when the two different discount factors are sufficiently large.

Pareto efficiency has not yet received enough attention in this type of model. In the bargaining model of Rubinstein (1982), it is obvious that every immediate agreement is also Pareto efficient. In the negotiation model with different time preferences, however, this is no longer the case. Making this more precise requires the insights of Lehrer and Pauzner (1999) in the context of repeated games and also the modification of their technique for the negotiation model. Lehrer and Pauzner (1999) show that any sequence of two payoff vectors may not lead to a convex combination of these two payoff vectors when the players have different time preferences. Pareto improvement can be realized if the less-patient player trades his long-run payoffs for short-run payoffs. ${ }^{5}$ They demonstrate that when two players have different time preferences, many SPE payoffs in a repeated game are not in the set of feasible and individually rational payoffs, as traditionally defined. In the negotiation model, such Pareto improvement is also present so that some feasible payoff vectors could be above the bargaining frontier. This implies that not every immediate agreement is Pareto efficient. As we will make clear later, what matters the most in characterizing the set of SPE payoffs is the Pareto frontier of SPE payoffs, which is quite complicated under different time preferences.

\section{The Sets of SPE Payoffs}

The key to characterize the set of SPE payoffs is to derive each player's lowest and highest SPE payoffs. We first provide a set of necessary and sufficient conditions for these extreme SPE payoffs in Section 3.1. In applying these conditions to derive the extreme SPE payoffs in the current period, we need the Pareto frontier of SPE payoffs in the following period. We then focus on these effective continuation payoffs in Section 3.2. It turns out that the

\footnotetext{
${ }^{5}$ The results in e.g. Ramsey (1928) and Bewley (1972) indicate that similar insights already have a long history in economics.
} 
Pareto frontier of SPE payoffs depends on both the discount factors and the extreme SPE payoffs. This inter-dependency between the extreme SPE payoffs and the Pareto frontier of SPE payoffs requires a new set of techniques to analyze the negotiation model with different time preferences. Unlike the case of common time preferences, no closed-form solutions for the extreme SPE payoffs are available in general. However, our analysis is sufficient to characterize the extreme SPE payoffs, and hence the set of SPE payoffs in any negotiation game. We will reexamine the case of common time preferences and tie our analysis to the existing literature on the negotiation model.

\subsection{Extreme SPE Payoffs}

Let $E^{i}$, for $i=1$ and 2 , be the set of SPE payoffs in any period in which player $i$ makes a proposal to player $j$ for $j \neq i$. For simplicity, we suppress all the other parameters that $E^{i}$ may depend on, such as the discount factors. Given the existence of a stationary SPE (Proposition 1) and the model setup, $E^{i}$ is a non-empty and bounded subset of $\mathbb{R}_{+}^{2}$. Applying the technique of self-generating payoffs for a repeated game by Abreu et al. (1986, 1990), and for a bargaining game by Shaked and Sutton (1984) and Binmore (1987), ${ }^{6}$ we can prove that $E^{i}$ is also compact and convex. ${ }^{7}$ Given the compactness of $E^{i}$ for $i=1$ and 2, player $l$ 's lowest and highest SPE payoffs when player $i$ makes a proposal are, respectively,

$$
m_{l}^{i}=\min _{v \in E^{i}} v_{l} \quad \text { and } \quad M_{l}^{i}=\max _{v \in E^{i}} v_{l} \quad \text { for } l=i \text { and } j
$$

In any period in which player $i$ makes a proposal, if player $j$ rejects player $i$ 's proposal then they will have to play a disagreement outcome $a \in A$ in the current period and a continuation SPE with payoff vector $v=\left(v_{i}, v_{j}\right) \in E^{j}$ in the following period in which player $j$ proposes. The continuation payoff vector generally depends on the disagreement outcome so that after a player deviates, the continuation payoff vector may change accordingly. Given the continuation payoff vector $v \in E^{j}$, playing $a \in A$ in the disagreement game $G$ is sequentially

\footnotetext{
${ }^{6}$ See e.g., Mailath and Samuelson (2006) and van Damme (1991) for more comprehensive treatments of self-generating sets of SPE payoffs.

${ }^{7}$ Upon request, a detailed proof is available from the authors.
} 
rational if and only if

$$
\left(1-\delta_{l}\right) d_{l}(a)+\delta_{l} v_{l} \geq\left(1-\delta_{l}\right) g_{l}(a)+\delta_{l} m_{l}^{j} \quad \text { for } l=i \text { and } j .
$$

Inequality (3) states that player $l$ 's payoff from complying is at least what he could obtain by deviating from $a \in A$ followed by his lowest SPE payoff in the following period. Obviously, any Nash equilibrium of $G$ satisfies (3) for all discount factors and all continuation payoffs. By incorporating the possibility of unacceptable proposals explicitly in the backward induction technique of Shaked and Sutton (1984), we obtain the following result:

Proposition 3 For all $\left(\delta_{i}, \delta_{j}\right) \in(0,1)^{2}$, we have

$$
\begin{aligned}
& m_{i}^{i}=\min _{a \in A, v \in E^{j}} \max \left\{\begin{array}{l}
\left(1-\delta_{i}\right) d_{i}(a)+\delta_{i} v_{i}, \\
1-\left(1-\delta_{j}\right) d_{j}(a)-\delta_{j} v_{j},
\end{array} \quad\right. \text { s.t. (3), } \\
& M_{i}^{i}=\max _{a \in A, v \in E^{j}} \max \left\{\begin{array}{l}
\left(1-\delta_{i}\right) d_{i}(a)+\delta_{i} v_{i}, \\
1-\left(1-\delta_{j}\right) d_{j}(a)-\delta_{j} v_{j},
\end{array} \quad\right. \text { s.t. (3), } \\
& m_{j}^{i}=\min _{a \in A, v \in E^{j}}\left(1-\delta_{j}\right) d_{j}(a)+\delta_{j} v_{j} \quad \text { s.t. (3), } \\
& M_{j}^{i}=\max _{a \in A, v \in E^{j}}\left(1-\delta_{j}\right) d_{j}(a)+\delta_{j} v_{j} \quad \text { s.t. (3). }
\end{aligned}
$$

Proof. Consider the general structure of any SPE in a period in which player $i$ proposes. Suppose player $i$ proposes $\hat{x}=\left(\hat{x}_{i}, \hat{x}_{j}\right) \in \Delta$, player $j$ either accepts or rejects $\hat{x}$, and the continuation SPE consists of $a(x) \in A$ and $v(x) \in E^{j}$ that satisfy (3) after player $j$ rejects player $i$ 's proposal $x$ for all $x \in \Delta$. Note that the continuation SPE generally depends on what proposal player $j$ rejects. Denote player $i$ 's payoff from such a SPE as

$$
v_{i}^{*}= \begin{cases}\left(1-\delta_{i}\right) d_{i}(a(\hat{x}))+\delta_{i} v_{i}(\hat{x}), & \text { if player } j \text { rejects } \hat{x} \\ 1-\hat{x}_{j}, & \text { if player } j \text { accepts } \hat{x} .\end{cases}
$$

First, consider any sequence of proposals $\left\{x^{n}\right\}_{n=1}^{\infty} \subset \Delta$ such that $x_{i}^{n}=1-x_{j}^{n}>v_{i}^{*}$ for all $n \geq 1$ and $\lim _{n \rightarrow \infty} x_{i}^{n}=v_{i}^{*}$. For $v_{i}^{*}$ to be player $i$ 's SPE payoff, player $j$ must reject $x^{n}$ and player $i$ must receive no more than $v_{i}^{*}$ after player $j$ rejects $x^{n}$ for all $n \geq 1$ (otherwise, player $i$ would have an incentive to deviate to propose $x^{n}$ instead). In other words, for all $n \geq 1$, we have

$$
\left(1-\delta_{i}\right) d_{i}\left(a\left(x^{n}\right)\right)+\delta_{i} v_{i}\left(x^{n}\right) \leq v_{i}^{*} \quad \text { and } \quad x_{j}^{n} \leq\left(1-\delta_{j}\right) d_{j}\left(a\left(x^{n}\right)\right)+\delta_{j} v_{j}\left(x^{n}\right)
$$


Since $A$ is compact by assumption, sequence $\left\{a\left(x^{n}\right)\right\}_{n=1}^{\infty} \subset A$ has a convergent subsequence, say (without loss of generality) $\lim _{n \rightarrow \infty} a\left(x^{n}\right)=\bar{a} \in A$. The compactness of $E^{j}$ then implies that $\left\{v\left(x^{n}\right)\right\}_{n=1}^{\infty} \subset E^{j}$ also has a convergent subsequence, say (without loss of generality) $\lim _{n \rightarrow \infty} v\left(x^{n}\right)=\bar{v}$. Since every $\left(a\left(x^{n}\right), v\left(x^{n}\right)\right)$ satisfies (3) for all $n \geq 1$, so does $(\bar{a}, \bar{v})$ due to the continuity of $d(\cdot)$. As $n \rightarrow \infty$, the two inequalities in (9) yield

$$
\left(1-\delta_{i}\right) d_{i}(\bar{a})+\delta_{i} \bar{v}_{i} \leq v_{i}^{*} \quad \text { and } \quad 1-v_{i}^{*} \leq\left(1-\delta_{j}\right) d_{j}(\bar{a})+\delta_{j} \bar{v}_{j} .
$$

The last two inequalities imply that

$$
v_{i}^{*} \geq \max \left\{\begin{array}{l}
\left(1-\delta_{i}\right) d_{i}(\bar{a})+\delta_{i} \bar{v}_{i}, \\
1-\left(1-\delta_{j}\right) d_{j}(\bar{a})-\delta_{j} \bar{v}_{j},
\end{array}\right.
$$

which is bounded from below by the right-hand side of (4). Let $(\hat{a}, \hat{v})$ be a solution to the optimization problem in (4). Consider the following strategy profile $\hat{\sigma}$ :

- player $i$ proposes $\hat{x}$ where $\hat{x}_{i}=\max \left\{\begin{array}{l}\left(1-\delta_{i}\right) d_{i}(\hat{a})+\delta_{i} \hat{v}_{i}, \\ 1-\left(1-\delta_{j}\right) d_{j}(\hat{a})-\delta_{j} \hat{v}_{j},\end{array}\right.$

— player $j$ accepts $x$ if and only if $x_{j} \geq\left(1-\delta_{j}\right) d_{j}(\hat{a})+\delta_{j} \hat{v}_{j}$, and

— the continuation SPE is $(a(x), v(x))=(\hat{a}, \hat{v})$ after player $j$ rejects $x$ for all $x \in \Delta$.

Strategy profile $\hat{\sigma}$ constitutes a SPE, from which player $i$ receives exactly (4). Hence, (4) characterizes player $i$ 's lowest SPE payoff $m_{i}^{i}$.

Second, accepting $\hat{x}$ is a best response for player $j$ if and only if $\hat{x}_{j} \geq\left(1-\delta_{j}\right) d_{j}(a(\hat{x}))+$ $\delta_{j} v_{j}(\hat{x})$. Together with (8), we have

$$
v_{i}^{*} \leq \begin{cases}\left(1-\delta_{i}\right) d_{i}(a(\hat{x}))+\delta_{i} v_{i}(\hat{x}), & \text { if player } j \text { rejects } \hat{x}, \\ 1-\left(1-\delta_{j}\right) d_{j}(a(\hat{x}))-\delta_{j} v_{j}(\hat{x}), & \text { if player } j \text { accepts } \hat{x},\end{cases}
$$

which is bounded from above by the right-hand side of (5). The strategy profile $\hat{\sigma}$ above with $(\hat{a}, \hat{v})$ being a solution to the optimization problem in (5) supports player $i$ 's highest SPE payoff $M_{i}^{i}$.

Third, player $j$ certainly rejects any $x \in \Delta$ such that $x_{j}$ is less than the right-hand side of (6) because player $i$ cannot receive less than his lowest continuation payoff after rejecting any proposal. Therefore, player $j$ 's SPE payoffs are bounded from below by the right-hand 
side of (6). Furthermore, player $j$ receives exactly the right-hand side of (6) in the SPE $\hat{\sigma}$ above with $(\hat{a}, \hat{v})$ being a solution to the optimization problem in (6).

Lastly, since player $j$ certainly accepts any $x \in \Delta$ such that $x_{j}$ is greater than the righthand side of (7), player $i$ will never propose $x \in \Delta$ such that $x_{j}$ is more than player $j$ 's highest continuation payoff. In other words, $\hat{x}_{j}$ must be less than or equal to the right-hand side of (7). Whether player $j$ accepts $\hat{x}$ or not, player $j$ cannot obtain more than the right-hand side of (7). Again, player $j$ receives exactly the right-hand side of (7) in the SPE $\hat{\sigma}$ above with $(\hat{a}, \hat{v})$ being a solution to the optimization problem in $(7)$.

Although the objective functions in the optimization problems of Proposition 3 are welldefined and continuous, $A$ is given and compact, we know nothing about $E^{j}$ at this stage other than its non-emptiness, compactness, and convexity. In order to fully understand the issues involved, we have to discuss effective continuation SPE payoffs in solving for these extreme SPE payoffs. We next show that the most effective continuation SPE payoffs to (4) - (7) are those that are on the Pareto frontier of $E^{j}$. Accordingly, denote the Pareto frontier of $E^{j}$ as

$$
\varphi^{j}\left(v_{i}\right)=\max _{v^{\prime} \in E^{j}} v_{j}^{\prime} \quad \text { s.t. } v_{i}^{\prime} \geq v_{i} \quad \text { and } \quad \varphi^{i}\left(v_{j}\right)=\max _{v^{\prime} \in E^{j}} v_{i}^{\prime} \quad \text { s.t. } v_{j}^{\prime} \geq v_{j} .
$$

Since $E^{j}$ is a non-empty, compact, and convex subset of $\mathbb{R}_{+}^{2}$, both $\varphi^{i}(\cdot)$ and $\varphi^{j}(\cdot)$ are continuous and non-increasing. Given Proposition 3, the following conditions on the responding player $j$ 's extreme SPE payoffs are immediate:

Proposition 4 For all $\left(\delta_{i}, \delta_{j}\right) \in(0,1)^{2}$, we have

$$
\begin{aligned}
m_{j}^{i} & \geq \delta_{j} m_{j}^{j} \\
M_{j}^{i} & \leq \max _{a \in A}\left[\left(1-\delta_{j}\right) d_{j}(a)+\delta_{j} \varphi^{j}\left(\frac{1-\delta_{i}}{\delta_{i}}\left[g_{i}(a)-d_{i}(a)\right]+\delta_{i} m_{i}^{i}\right)\right] .
\end{aligned}
$$

Proof. Substituting (3) into (6), we have

$$
m_{j}^{i} \geq \min _{a \in A}\left[\left(1-\delta_{j}\right) g_{j}(a)+\delta_{j} m_{j}^{j}\right]=\left(1-\delta_{j}\right) \min _{a \in A} g_{j}(a)+\delta_{j} m_{j}^{j}=\delta_{j} m_{j}^{j},
$$


which is (11). Notice that player $j$ 's minimax value $\min _{a} g_{j}(a)=0$ by assumption. For $l=j$, (3) and (10) imply that

$$
v_{j} \leq \varphi^{j}\left(v_{i}\right) \leq \varphi^{j}\left(\frac{1-\delta_{i}}{\delta_{i}}\left[g_{i}(a)-d_{i}(a)\right]+m_{i}^{j}\right) .
$$

Substituting (13) into (7), we obtain (12).

For sufficiently large $\left(\delta_{i}, \delta_{j}\right) \in(0,1)^{2}$, Proposition 4 implicitly specifies how the players behave in the responding player's worst and best SPE. In player $j$ 's worst SPE, if player $j$ rejects any proposal, he will receive his minimax value of 0 during the current disagreement period followed by his lowest SPE payoff $m_{j}^{j}$ in the following period. In player $j$ 's best SPE, on the other hand, if player $j$ rejects any proposal, he will receive his highest continuation payoff, provided that player $i$ will be compensated in the following period. In fact, when the players are sufficiently patient, (11) and (12) hold with equalities for the responding player's lowest and highest SPE payoffs. These results are similar to those of Busch and Wen (1995), where the players have the same discount factor.

We now turn to the proposing player's extreme SPE payoffs.

Proposition 5 For all $\left(\delta_{i}, \delta_{j}\right) \in(0,1)^{2}$, we have

$$
\begin{aligned}
& m_{i}^{i} \geq \min _{a \in A} \max \left\{\begin{array}{l}
\left(1-\delta_{i}\right) g_{i}(a)+\delta_{i}^{2} m_{i}^{i}, \\
1-\left(1-\delta_{j}\right) d_{j}(a)-\delta_{j} \varphi^{j}\left(\frac{1-\delta_{i}}{\delta_{i}}\left[g_{i}(a)-d_{i}(a)\right]+\delta_{i} m_{i}^{i}\right),
\end{array}\right. \\
& M_{i}^{i} \leq \max \left\{\begin{array}{l}
\max _{a \in A}\left[\left(1-\delta_{i}\right) d_{i}(a)+\delta_{i} \varphi^{i}\left(\frac{1-\delta_{j}}{\delta_{j}}\left[g_{j}(a)-d_{j}(a)\right]+m_{j}^{j}\right)\right], \\
1-\delta_{j} m_{j}^{j} .
\end{array}\right.
\end{aligned}
$$

Proof. With Proposition 4, substituting (3) and (13) into (4) yields (14);

$$
\begin{aligned}
m_{i}^{i} \geq \min _{a \in A} \max \left\{\begin{array}{l}
\left(1-\delta_{i}\right) g_{i}(a)+\delta_{i} m_{i}^{j}, \\
1-\left(1-\delta_{j}\right) d_{j}(a)-\delta_{j} \varphi^{j}\left(\frac{1-\delta_{i}}{\delta_{i}}\left[g_{i}(a)-d_{i}(a)\right]+m_{i}^{j}\right),
\end{array}\right. \\
\geq \min _{a \in A} \max \left\{\begin{array}{l}
\left(1-\delta_{i}\right) g_{i}(a)+\delta_{i}^{2} m_{i}^{i}, \\
1-\left(1-\delta_{j}\right) d_{j}(a)-\delta_{j} \varphi^{j}\left(\frac{1-\delta_{i}}{\delta_{i}}\left[g_{i}(a)-d_{i}(a)\right]+\delta_{i} m_{i}^{i}\right) .
\end{array}\right.
\end{aligned}
$$

For $l=i,(3)$ and (10) imply that

$$
v_{i} \leq \varphi^{i}\left(v_{j}\right) \leq \varphi^{i}\left(\frac{1-\delta_{j}}{\delta_{j}}\left[g_{j}(a)-d_{j}(a)\right]+m_{j}^{j}\right) .
$$


Substituting the last inequality and (3) into (7) yields (4);

$$
\begin{aligned}
M_{i}^{i} & \leq \max \left\{\begin{array}{l}
\max _{a}\left[\left(1-\delta_{i}\right) d_{i}(a)+\delta_{i} \varphi^{i}\left(\frac{1-\delta_{j}}{\delta_{j}}\left[g_{j}(a)-d_{j}(a)\right]+m_{j}^{j}\right)\right] \\
\max _{a}\left[1-\left(1-\delta_{j}\right) g_{j}(a)-\delta_{j} m_{j}^{j}\right]
\end{array}\right. \\
& =\max \left\{\begin{array}{l}
\max _{a}\left[\left(1-\delta_{i}\right) d_{i}(a)+\delta_{i} \varphi^{i}\left(\frac{1-\delta_{j}}{\delta_{j}}\left[g_{j}(a)-d_{j}(a)\right]+m_{j}^{j}\right)\right], \\
1-\delta_{j} m_{j}^{j}
\end{array}\right.
\end{aligned}
$$

where the last equation is due to the assumption that $\min _{a} g_{j}(a)=0$.

Given the Pareto frontier of $E^{j}$, Propositions 4 and 5 imply that $m_{i}^{i}$ and $m_{j}^{j}$ are essential to determine the other extreme SPE payoffs. For the benchmark $\delta_{i}=\delta_{j}=\delta$, the Pareto frontier of SPE payoffs coincides with the bargaining frontier, i.e., $\varphi^{j}\left(v_{i}\right)=1-v_{i}$. Consequently, (14) simplifies to

$$
\begin{aligned}
m_{i}^{i} & \geq \min _{a \in A} \max \left\{\begin{array}{l}
(1-\delta) g_{i}(a)+\delta^{2} m_{i}^{i}, \\
1-(1-\delta) d_{j}(a)-\delta\left(1-\frac{1-\delta}{\delta}\left[g_{i}(a)-d_{i}(a)\right]+\delta m_{i}^{i}\right),
\end{array}\right. \\
& =\min _{a \in A} \max \left\{\begin{array}{l}
(1-\delta) g_{i}(a) \\
(1-\delta)\left[1-d_{i}(a)-d_{j}(a)+g_{i}(a)\right]
\end{array}+\delta^{2} m_{i}^{i},\right. \\
& =(1-\delta) \min _{a \in A}\left[1-d_{i}(a)-d_{j}(a)+g_{i}(a)\right]+\delta^{2} m_{i}^{i},
\end{aligned}
$$

due to $1-d_{i}(a)-d_{j}(a) \geq 0$ for all $a \in A$. From (16), we have

$$
m_{i}^{i} \geq \frac{1}{1+\delta}\left[1-\max _{a \in A}\left[d_{i}(a)-d_{j}(a)+g_{i}(a)\right]\right],
$$

which is the key step to establish Proposition 2. When $\delta_{i} \neq \delta_{j}$, we need to discuss the Pareto frontier of $E^{j}$, i.e., effective continuation payoff in order to solve $m_{i}^{i}$ from (14).

\subsection{Effective Continuation SPE Payoffs}

In order to solve $m_{i}^{i}$ from Proposition 3, we need to discuss first how $E^{j}$ is determined by the players' lowest SPE payoffs. Whenever player $j$ proposes, outcome path $\pi=\left(a^{1}, a^{2}, \cdots, a^{T}, x\right)$ can be supported as a SPE outcome path if and only if for all $t \leq T+1$,

$$
\left(1-\delta_{l}\right) \sum_{s=t}^{T} \delta_{l}^{s-t} d_{l}\left(a^{s}\right)+\delta_{l}^{T+1-t} x_{l} \geq\left\{\begin{array}{ll}
m_{l}^{i}, & \text { if } t \text { is even, } \\
m_{l}^{j}, & \text { if } t \text { is odd, }
\end{array} \quad \text { for } l=i \text { and } j,\right.
$$

and for all $t \leq T$,

$$
\left(1-\delta_{l}\right) \sum_{s=t}^{T} \delta_{l}^{s-t} d_{l}\left(a^{s}\right)+\delta_{l}^{T+1-t} x_{l} \geq\left(1-\delta_{l}\right) g_{l}\left(a^{t}\right)+ \begin{cases}\delta_{l} m_{l}^{j}, & \text { if } t \text { is even, } \\ \delta_{l} m_{l}^{i}, & \text { if } t \text { is odd. }\end{cases}
$$


For $t=T+1$, (17) implies that no matter who proposes the final agreement $x \in \Delta$, it needs to be a SPE agreement in period $T+1$. (18) states that if player $l$ deviates from $a^{t}$ in period $t \leq T$, then player $l$ will be punished by his lowest SPE payoff, either $m_{l}^{i}$ or $m_{l}^{j}$, in the following period. With publicly correlated strategies, $E^{j}$ is the convex hull of $\{v(\pi):(17)$ and (18) $\}$. Note that with $T=0,(17)$ and (18) imply that any immediate agreement $\left(x_{i}, 1-x_{i}\right)$ for $x_{i} \in\left[m_{i}^{j}, 1-m_{j}^{j}\right]$ belongs $E^{j} .{ }^{8}$ Hence, $\varphi^{j}\left(v_{i}\right) \geq 1-v_{i}$ for all $\left(\delta_{i}, \delta_{j}\right) \in(0,1)^{2}$ and $v_{i} \in\left[m_{i}^{j}, 1-m_{j}^{j}\right]$.

Due to Proposition 4, when the discount factors are sufficiently large, we can rewrite (17) and (18) in terms of $m_{i}^{i}$ and $m_{j}^{j}$ only. Consequently, the set $E^{j}$ depends on $m_{i}^{i}$ and $m_{j}^{j}$ only. For $i=1$ and 2 , substituting $E^{j}(j \neq i)$ in terms of $m_{i}^{i}$ and $m_{j}^{j}$ into (4) provides two equations, one for $m_{1}^{1}$ and one for $m_{2}^{2}$. The solution $m_{i}^{i}$ from such an implicit equation system can be supported as player $i$ 's (lowest) SPE payoff for $i=1$ and 2 when the discount factors are sufficiently large.

In Proposition 5, we show that only the Pareto frontier of $E^{j}$ is effective in solving $m_{i}^{i}$ from (14). In the rest of this subsection, we provide specific structures on the continuation paths that achieve the Pareto frontier of $E^{j}$. When $\delta_{i}=\delta_{j}$, the Pareto frontier of $E^{j}$ is a connected segment of the unit simplex. When $\delta_{i} \neq \delta_{j}$, as in a repeated game with different time preferences, many SPE payoffs in a negotiation game can be above the bargaining frontier. Lehrer and Pauzner (1999) investigate in great detail the Pareto frontier of SPE payoffs in a repeated game under different time preferences. There are many obstacles in directly applying their results to a negotiation game. SPE payoffs in a repeated game are bounded from below by players' stage-game minimax payoffs that are invariant with respect to the discount factors and time periods. In a negotiation game, however, players' lowest SPE payoffs depend on the discount factors and also who proposes. A typical outcome path in a negotiation game ends with an agreement that ceases any future payoff variation. In a repeated game, it may not possible to have a SPE in which a player receives exactly his minimax payoff, so it is often sufficient to provide a SPE where a player's payoff is sufficiently

\footnotetext{
${ }^{8}$ This is the range of SPE payoffs with immediate agreement in Haller and Holden (1990).
} 
close to his minimax value. In a negotiation game, however, we need the SPE where a player receives exactly his lowest SPE payoff. In order to derive the Pareto frontier of $E^{j}$, we have to modify Lehrer and Pauzner's technique for these differences between a repeated game and a negotiation game.

According to Lehrer and Pauzner (1999), in order to characterize the Pareto frontier of $E^{j}$ in the direction of $\lambda=\left(\lambda_{i}, \lambda_{j}\right) \in \Delta$, we need to solve the following optimization problem:

$$
\max _{\pi} \lambda \cdot v(\pi), \quad \text { subject to }(17) \text { and }(18) .
$$

In other words, (19) provides the payoff vectors on the Pareto frontier $E^{j}$ in the direction of $\lambda \in \Delta$. When $G$ is a finite game, $E^{j}$ is a polygon in $\mathbb{R}_{+}^{2}$ and (19) provides us all the vertices in the direction of $\lambda \in \Delta$. Note that under (17) and (18), we can write (19) as

$$
\max _{T \geq 0} \sum_{t=1}^{\infty} \max _{y^{t}}\left[\lambda_{i}\left(1-\delta_{i}\right) \delta_{i}^{t-1} y_{i}+\lambda_{j}\left(1-\delta_{j}\right) \delta_{j}^{t-1} y_{j}\right]
$$

where $y^{t}=d\left(a^{t}\right) \in d(A)$ for all $t \leq T$ and $y^{t}=x \in \Delta$ for all $t>T$. In the rest of this subsection, we will solely focus on the case $\delta_{i}<\delta_{j}$, while similar arguments apply when $\delta_{i}>\delta_{j}$. When $\delta_{i}<\delta_{j}$, for all $\lambda=\left(\lambda_{i}, \lambda_{j}\right) \in \Delta$, the weight ratio

$$
\frac{\lambda_{j}\left(1-\delta_{j}\right) \delta_{j}^{t-1}}{\lambda_{i}\left(1-\delta_{i}\right) \delta_{i}^{t-1}}
$$

is monotonically increasing with respect to $t \geq 0$. Therefore, in any potential solution to (19) with $T>0$, we must have

$$
d_{i}\left(a^{t}\right) \geq d_{i}\left(a^{t+1}\right)>x_{i} \quad \text { and } \quad d_{j}\left(a^{t}\right) \leq d_{j}\left(a^{t+1}\right)<x_{j}=1-x_{i}
$$

whenever it is possible under (17) and (18). Given $\delta_{i}<\delta_{j}$, the weight ratio will be greater than one for sufficiently large $t$. This implies that two player must reach an agreement within finite periods in any potential solution to (19). These arguments narrow down the potential solutions to (19). Given (20), sequential rationality (17) simplifies to $x_{i} \geq m_{i}^{i}$ if $T$ is even, and $x_{i} \geq m_{i}^{j}$ if $T$ is odd. For all $t \leq T, d\left(a^{t}\right)$ should be as close as possible to the Pareto frontier of $d(A)$, provided that (17) and (18) hold. As in a repeated game, player $j$ 's 


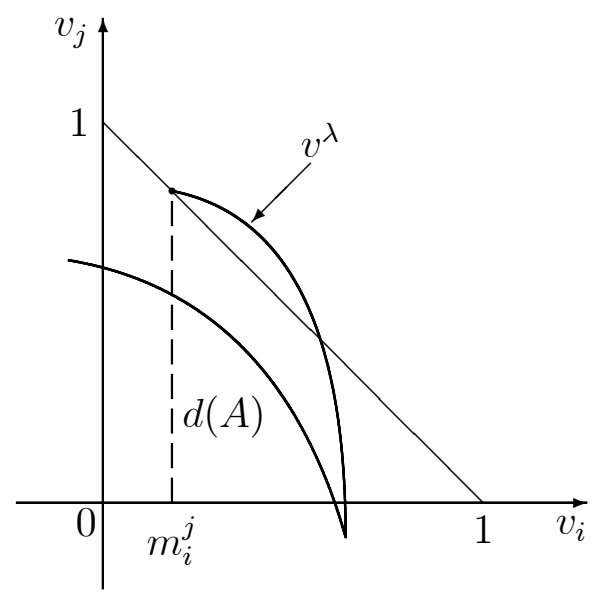

Figure 1: The curve of payoff vectors $v^{\lambda}$ for all $\lambda \in \Delta$.

per-period payoff during the early phase of such an outcome path could be lower than his minimax value. Since player $j$ 's payoff increases over time, his average payoff from the entire path will not be less than his lowest SPE payoffs. Let $v^{\lambda}$, for all $\lambda \in \Delta$, denote the payoff vector resulting from a solution to (19) under (20). Figure 1 illustrates the curve of payoff vectors $v^{\lambda}$ for all $\lambda \in \Delta$. Similar as in Lehrer and Pauzner (1999), this curve is continuous in $\lambda$ and all payoff vectors $\left(m_{i}^{i}, 1-m_{i}^{i}\right)$ and $d\left(a^{t}\right)$.

Figure 1 does not fully specify the Pareto frontier of $E^{j}$, because the outcome path $\pi=(\tilde{x})$ with an immediate agreement $\tilde{x}_{j}=m_{j}^{j}$ (with $T=0$ ) may also solve (19), in particular when $\lambda_{j} / \lambda_{i}$ is sufficiently close to 0 . With publicly correlated strategies, the Pareto frontier of $E^{j}$ is completely characterized by $\tilde{x}$ and $v^{\lambda}$ for all $\lambda \in \Delta$. Figure 2 illustrates the two possible cases. Given $m_{j}^{j}$, $E^{j}$ (as a correspondence of $m_{i}^{i}$ ) is convex-valued and continuous with respect to $m_{i}^{i}$.

To summarize, whenever time preferences are sufficiently different, the Pareto frontier of $E^{j}$ is generally above the bargaining frontier. This will affect how $m_{i}^{i}$ is determined. As we have shown above, the Pareto frontier of $E^{j}$ is rather complicated, which prevents us from obtaining a closed-form solution for $m_{i}^{i}$. Nevertheless, our analysis provides a general technique on how to solve the players' lowest SPE payoffs, and, hence, how to characterize 


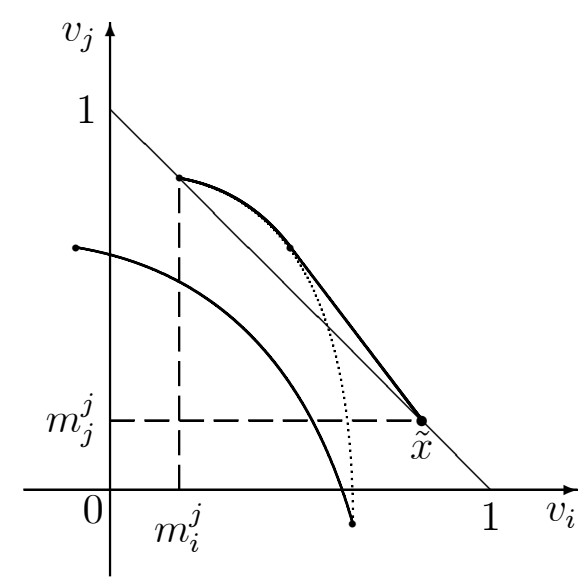

(a) $\tilde{x}$ is Pareto efficient

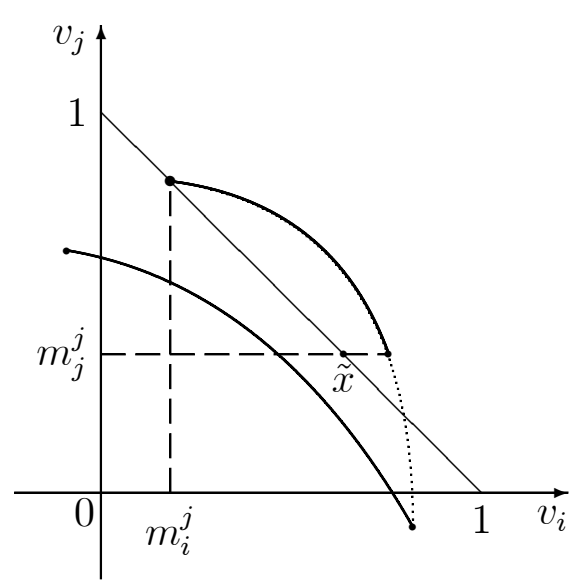

(b) $\tilde{x}$ is not Pareto efficient

Figure 2: The Pareto frontier of $E^{j}$ where $\tilde{x}=\left(1-m_{j}^{j}, m_{j}^{j}\right)$.

the set of SPE payoffs in a negotiation game when $\delta_{i} \neq \delta_{j}$. In the next section, we will demonstrate how this technique works for common interest disagreement games.

\subsection{Proposing Player's Lowest SPE Payoff}

In the previous subsections, we established that $m_{i}^{i}$ and $m_{j}^{j}$ are the key in characterizing the other extreme SPE payoffs. Note that condition (14) depends on $m_{i}^{i}$ directly and on $m_{i}^{i}$ and $m_{j}^{j}$ indirectly. In this subsection, we will show how to solve $m_{i}^{i}$ as the least fixed point to (14). Figure 2 indicates that we need to analyze the following two distinct cases:

Case 1: $\delta_{i}<\delta_{j}$

Instead of solving $m_{i}^{i}$ and $m_{j}^{j}$ simultaneously from the implicit equation system implied by (14), we can first find $m_{i}^{i}$ independently of $m_{j}^{j}$ when $\delta_{i}$ and $\delta_{j}$ are sufficiently large. Condition (14) can be rewritten as $m_{i}^{i} \geq \Lambda\left(m_{i}^{i}\right)$, where

$$
\Lambda\left(m_{i}^{i}\right)=\min _{a \in A} \max \left\{\begin{array}{l}
\left(1-\delta_{i}\right) g_{i}(a)+\delta_{i}^{2} m_{i}^{i}, \\
1-\left(1-\delta_{j}\right) d_{j}(a)-\delta_{j} \varphi^{j}\left(\delta_{i} m_{i}^{i}+\frac{1-\delta_{i}}{\delta_{i}}\left[g_{i}(a)-d_{i}(a)\right]\right) .
\end{array}\right.
$$

Since $\varphi^{j}\left(\delta_{i} m_{i}^{i}+\frac{1-\delta_{i}}{\delta_{i}}\left[g_{i}(a)-d_{i}(a)\right]\right)$ is continuous with respect to $a \in A, m_{i}^{i}$ and (indirectly) $m_{j}^{j},(21)$ is a well-defined minimax problem because the right-hand side is continuous in $m_{i}^{i}$ and (indirectly) $m_{j}^{j}$. To solve $(21)$ when $\delta_{i}$ is sufficiently close to 1 , we only need to know 
$\varphi^{j}\left(v_{i}\right)$ for $v_{i}$ sufficiently close to $\delta_{i} m_{i}^{i}$, i.e.,

$$
v_{i} \in\left[\delta_{i} m_{i}^{i}, \delta_{i} m_{i}^{i}+\frac{1-\delta_{i}}{\delta_{i}} \max _{a \in A}\left[g_{i}(a)-d_{i}(a)\right]\right] .
$$

As we have shown in Section 3.2 for the case of $\delta_{i}<\delta_{j}, \varphi^{j}(\cdot)$ in this part of its domain will be independent of $m_{j}^{j}$ for sufficiently large $\left(\delta_{i}, \delta_{j}\right) \cdot{ }^{9}$ Consequently, we have

Proposition 6 For sufficiently large $\left(\delta_{i}, \delta_{j}\right) \in(0,1)^{2}$ and $\delta_{i}<\delta_{j}, m_{i}^{i}$ is bounded from below by the least fixed point of $\Lambda(\cdot)$ in (21) and $\Lambda(\cdot)$ admits a fixed point in $\left[0, \hat{m}_{i}^{i}\right]$, where

$$
\hat{m}_{i}^{i}=\min _{a \in A} \max \left\{\begin{array}{l}
\frac{g_{i}(a)}{1+\delta_{i}}, \\
\frac{1-\delta_{j}}{1-\delta_{i} \delta_{j}}\left[1-d_{j}(a)+\frac{\delta_{j}}{\delta_{i}} \frac{1-\delta_{i}}{1-\delta_{j}}\left[g_{i}(a)-d_{i}(a)\right]\right] .
\end{array}\right.
$$

Proof. Since $\varphi^{j}(\cdot)$ is continuous and monotonically decreasing in $m_{i}^{i}$ (both directly and indirectly), and independent of $m_{j}^{j}, \Lambda(\cdot)$ is a well-defined, continuous and monotonically increasing function of $m_{i}^{i}$ only. Note that from (21), we have $\Lambda(0) \geq\left(1-\delta_{i}\right) \min _{a \in A} g_{i}(a)=0$. Let $\hat{a}$ be a solution to (22). Evaluating the right-hand side of (21) at $\hat{m}_{i}^{i}$ and $\hat{a}$, we have

$$
\begin{aligned}
\Lambda\left(\hat{m}_{i}^{i}\right) & \leq \max \left\{\begin{array}{l}
\left(1-\delta_{i}\right) g_{i}(\hat{a})+\delta_{i}^{2} \hat{m}_{i}^{i} \\
1-\left(1-\delta_{j}\right) d_{j}(\hat{a})-\delta_{j} \varphi^{j}\left(\delta_{i} m_{i}^{i}+\frac{1-\delta_{i}}{\delta_{i}}\left[g_{i}(\hat{a})-d_{i}(\hat{a})\right]\right)
\end{array}\right. \\
& \leq \max \left\{\begin{array}{l}
\left(1-\delta_{i}\right) g_{i}(\hat{a})+\delta_{i}^{2} \hat{m}_{i}^{i} \\
\left(1-\delta_{j}\right)\left[1-d_{j}(\hat{a})-\frac{\delta_{j}}{\delta_{i}} \frac{1-\delta_{i}}{1-\delta_{j}}\left[g_{i}(\hat{a})-d_{i}(\hat{a})\right]\right]-\delta_{i} \delta_{j} \hat{m}_{i}^{i}
\end{array}\right.
\end{aligned}
$$

Notice that

$$
\begin{aligned}
\hat{m}_{i}^{i} & \geq \frac{1}{1+\delta_{i}} g_{i}(\hat{a}) \Leftrightarrow\left(1-\delta_{i}\right) g_{i}(\hat{a})+\delta_{i}^{2} \hat{m}_{i}^{i} \leq \hat{m}_{i}^{i}, \\
\hat{m}_{i}^{i} & \geq \frac{1-\delta_{j}}{1-\delta_{i} \delta_{j}}\left[1-d_{j}(\hat{a})-\frac{\delta_{j}}{\delta_{i}} \frac{1-\delta_{i}}{1-\delta_{j}}\left[g_{i}(\hat{a})-d_{i}(\hat{a})\right]\right] \\
& \Leftrightarrow\left(1-\delta_{j}\right)\left[1-d_{j}(\hat{a})-\frac{\delta_{j}}{\delta_{i}} \frac{1-\delta_{i}}{1-\delta_{j}}\left[g_{i}(\hat{a})-d_{i}(\hat{a})\right]\right]-\delta_{i} \delta_{j} \hat{m}_{i}^{i} \leq \hat{m}_{i}^{i} .
\end{aligned}
$$

Substituting the last two inequalities into (23) yields that $\Lambda\left(\hat{m}_{i}^{i}\right) \leq \hat{m}_{i}^{i}$. Due to its monotonicity, $\Lambda(\cdot)$ maps from $\left[0, \hat{m}_{i}^{i}\right]$ into itself. By Brouwer's fixed point theorem, $\Lambda(\cdot)$ has at least one fixed point in $\left[0, \hat{m}_{i}^{i}\right]$. Since $\Lambda(\cdot)$ is monotonically increasing, any value of $m_{i}^{i}$ that is strictly less than the least fixed point of $\Lambda(\cdot)$ certainly violates (14). This concludes the proof of the proposition.

\footnotetext{
${ }^{9}$ In fact, this part of $\varphi^{j}(\cdot)$ is the curve of vectors $v^{\lambda}$ in Figure 1, which is independent of $m_{j}^{j}$.
} 
The value of $\hat{m}_{i}^{i}$ can be tied to the existing literature: It is the maximum of the noconcession payoff in Bolt (1995) and the bound in Muthoo (1999) under the restriction that all continuation payoffs are bounded by the bargaining frontier. In Section 4, we show that $m_{i}^{i}<\hat{m}_{i}^{i}$ for a robust subclass of negotiation games.

Our next proposition asserts that when the discount factors are sufficiently large, the least fixed point of $\Lambda(\cdot)$ can be supported as player $i$ 's SPE payoff. Therefore, $m_{i}^{i}$ is indeed the least fixed point of $\Lambda(\cdot)$ when the discount factors are sufficiently large. Since the proof is rather long, we defer it to the appendix.

Proposition 7 There exists a $\hat{\delta} \in(0,1)$ such that for all $\delta_{j}>\delta_{i} \geq \hat{\delta}$, there is a SPE in which player $i$ receives the least fixed point of $\Lambda(\cdot)$.

Case 2: $\delta_{i}>\delta_{j}$

First, we solve $m_{j}^{j}$ independently as we described in Case 1 by switching $i$ and $j$. Once the value of $m_{j}^{j}$ is given, $\varphi^{j}\left(\delta_{i} m_{i}^{i}+\frac{1-\delta_{i}}{\delta_{i}}\left[g_{i}(a)-d_{i}(a)\right]\right)$ is a continuous function of $a \in A$ and $m_{i}^{i}$ only. This allows us to establish similar results for $m_{i}^{i}$ as in Proposition 6 and 7 for this case, which we omit. The proofs of Propositions 6 and 7 do not rely on the fact that $\delta_{i}<\delta_{j}$, except by requiring that $m_{i}^{i}$ be the only unknown variable in the part of $\varphi^{j}(\cdot)$ we need. Similar to the case of $\delta_{i}<\delta_{j}, m_{i}^{i}$ is the least fixed point of (21) for large enough $\left(\delta_{i}, \delta_{j}\right) \in(0,1)^{2}$. This ends our discussion on Case 2.

To conclude this section, we have provided a complete procedure to derive the proposing player's lowest SPE payoff for sufficiently large discount factors. We are then able to characterize other extreme SPE payoffs and, hence, the set of SPE payoffs in the negotiation model, as characterized in (17) and (18), when the discount factors are sufficiently large. Unlike the case of common time preferences, the Pareto frontier of SPE payoffs is rather complicated under different time preferences, which makes it impossible to obtain a closed-form solution to the proposing player's lowest SPE payoff. 


\section{Common Interest Disagreement Games}

In this section, we focus on an important class of negotiation games that contains the models studied in Fernandez and Glazer (1991), Haller and Holden (1990), Bolt (1995), and Slantchev (2003). In this case, the disagreement game is a common interest game where there exists a unique Pareto dominant disagreement outcome. Formally, there is an $a^{*} \in A$ such that $d\left(a^{*}\right) \geq d(a)$ for all $a \in A$. Without loss of too much generality, we assume that $d\left(a^{*}\right)$ is on the bargaining frontier $\Delta$, i.e., $d_{1}\left(a^{*}\right)+d_{2}\left(a^{*}\right)=1$. Obviously, $a^{*} \in A$ is a Nash equilibrium in $G$. When the players have a common discount factor $\delta \in(0,1)$, Proposition 2 asserts that for sufficiently large $\delta$, player $i$ 's lowest SPE payoff is equal to

$$
\begin{array}{ll}
\frac{1}{1+\delta}\left[1-d_{j}\left(a^{*}\right)\right]=\frac{d_{i}\left(a^{*}\right)}{1+\delta} & \text { when player } i \text { proposes and } \\
\frac{\delta}{1+\delta}\left[1-d_{j}\left(a^{*}\right)\right]=\frac{\delta d_{i}\left(a^{*}\right)}{1+\delta} & \text { when player } j \text { proposes. }
\end{array}
$$

Similar to player $i$ 's worst SPE in the case of common time preferences, it is not difficult to establish the following result:

Proposition 8 For sufficiently large $\left(\delta_{i}, \delta_{j}\right) \in(0,1)^{2}$, in the negotiation model with common interest disagreement games, there exists a SPE where player $i$ receives

$$
\begin{array}{ll}
\max \left\{\frac{1}{1+\delta_{i}} d_{i}\left(a^{*}\right), \frac{1-\delta_{j}}{1-\delta_{i} \delta_{j}}\left[1-d_{j}\left(a^{*}\right)\right]\right\} & \text { when } i \text { proposes and, } \\
\max \left\{\frac{\delta_{i}}{1+\delta_{i}} d_{i}\left(a^{*}\right), \frac{\delta_{i}\left(1-\delta_{j}\right)}{1-\delta_{i} \delta_{j}}\left[1-d_{j}\left(a^{*}\right)\right]\right\} & \text { when } j \text { proposes. }
\end{array}
$$

The SPE strategy profile specifies $a^{*}$ after player $i$ 's proposal is rejected and player $i$ 's minimax outcome in $G$ after player $i$ rejects any proposal by player $j$. In this SPE, player $i$ makes unacceptable proposals if and only if $\delta_{i}<\delta_{j}$. Despite the resemblance between Proposition 8 and Proposition 2, the SPE in Proposition 8 is generally not player $i$ 's worst $\mathrm{SPE}$ when $\delta_{i}$ and $\delta_{j}$ are significantly different. In order to characterize a player's worst SPE, we need to apply the analysis in Section 3 to this important class of negotiation games. 
With common interest disagreement games, most results in Section 3 can be further refined and simplified. First, $(7)$ implies that for all $\left(\delta_{i}, \delta_{j}\right) \in(0,1)^{2}$,

$$
M_{j}^{i} \leq\left(1-\delta_{j}\right) \max _{a \in A} d_{j}(a)+\delta_{j} \max _{v \in E^{j}} v_{j}=\left(1-\delta_{j}\right) d_{j}\left(a^{*}\right)+\delta_{j} M_{j}^{j}
$$

For sufficiently large $\left(\delta_{i}, \delta_{j}\right) \in(0,1)^{2},(24)$ implies that in player $j$ 's best SPE, if player $j$ rejects any proposal, player $j$ will receive his highest disagreement payoff $d_{j}\left(a^{*}\right)$ in the current period followed by his highest SPE payoff $M_{j}^{j}$ in the following period. Likewise, for sufficiently large $\left(\delta_{i}, \delta_{j}\right) \in(0,1)^{2}$, we have

$$
M_{i}^{i} \leq 1-m_{j}^{i}
$$

From (5), we obtain

$$
\begin{aligned}
M_{i}^{i} & =\max \begin{cases}\max _{a, v}\left[\left(1-\delta_{i}\right) d_{i}(a)+\delta_{i} v_{i}\right], & \text { s.t. }(3), \\
\max _{a, v}\left[1-\left(1-\delta_{j}\right) d_{j}(a)-\delta_{j} v_{j}\right], & \text { s.t. }(3),\end{cases} \\
& \leq \max \left\{\begin{array}{l}
\left(1-\delta_{i}\right) \max _{a \in A} d_{i}(a)+\delta_{i} \max _{v \in E^{j}} v_{i}, \\
1-\min _{a, v}\left[\left(1-\delta_{j}\right) d_{j}(a)-\delta_{j} v_{j}\right],
\end{array}\right. \\
& =\max \left\{\begin{array}{l}
\left(1-\delta_{i}\right) d_{i}\left(a^{*}\right)+\delta_{i} M_{i}^{j}, \\
1-m_{j}^{i} .
\end{array}\right.
\end{aligned}
$$

For sufficiently large $\left(\delta_{i}, \delta_{j}\right) \in(0,1)^{2}$, however, it cannot be the case that

$$
1-m_{j}^{i} \leq\left(1-\delta_{i}\right) d_{i}\left(a^{*}\right)+\delta_{i} M_{i}^{j}
$$

Suppose not, then $M_{i}^{i} \leq\left(1-\delta_{i}\right) d_{i}\left(a^{*}\right)+\delta_{i} M_{i}^{j}$ and (24) would imply that $M_{i}^{i} \leq d_{i}\left(a^{*}\right)$, which contradicts the fact that

$$
M_{i}^{i} \geq 1-\max \left\{\frac{1}{1+\delta_{j}} d_{j}\left(a^{*}\right), \frac{1-\delta_{i}}{1-\delta_{i} \delta_{j}}\left[1-d_{i}\left(a^{*}\right)\right]\right\}>d_{i}\left(a^{*}\right)
$$

implied by Proposition 8. Consequently, (25) must prevail. To summarize, inequalities (11), (24), and (25) provide us three of the four conditions to solve the extreme SPE payoffs. With common interest disagreement games, these three inequalities are relatively simple because they are not affected by the complications of the Pareto frontier of SPE payoffs. Condition (25) shows that Case (b) in Figure 2 is impossible when the disagreement is a common interest game. 
Condition (14) for $m_{i}^{i}$, which turns out to be the most important and most complicated piece of the puzzle, generally depends on the Pareto frontier of $E^{j}$. With common interest disagreement games, our next proposition provides a lower bound for $m_{i}^{i}$.

Proposition 9 For all $\left(\delta_{i}, \delta_{j}\right) \in(0,1)^{2}$, we have

$$
m_{i}^{i} \geq \frac{1-\delta_{j}}{1-\delta_{i} \delta_{j}}\left[1-d_{j}\left(a^{*}\right)\right] .
$$

Proof. Since $d_{j}(a) \leq d_{j}\left(a^{*}\right)$ for all $a \in A$ and $v_{j} \leq M_{j}^{j}$ for all $v \in E^{j}$, (25) and (11) imply that $M_{j}^{j} \leq 1-\delta_{i} m_{i}^{i}$. From (4), we have

$$
\begin{aligned}
m_{i}^{i} & \geq \min _{a \in A, v \in E^{j}} 1-\left(1-\delta_{j}\right) d_{j}(a)-\delta_{j} v_{j}, \quad \text { s.t. (3), } \\
& \geq 1-\left(1-\delta_{j}\right) d_{j}\left(a^{*}\right)-\delta_{j} M_{j}^{j} \\
& \geq 1-\left(1-\delta_{j}\right) d_{j}\left(a^{*}\right)-\delta_{j}\left(1-\delta_{i} m_{i}^{i}\right) \\
& =\left(1-\delta_{j}\right)\left[1-d_{j}\left(a^{*}\right)\right]+\delta_{j} \delta_{i} m_{i}^{i},
\end{aligned}
$$

which leads to the stated result.

Propositions 9 is rather important; it implies that for $\delta_{i} \geq \delta_{j}$, the SPE of Proposition 8 is indeed player $i$ 's worst SPE in this class of negotiation games. It extends player $i$ 's worst $\mathrm{SPE}$ in the case of common time preferences.

In the rest of this section, we will focus on the case that player $i$ is less patient than player $j ; \delta_{i}<\delta_{j}$. With common interest disagreement games, any effective continuation path that solves (19) must be generated from the following type of paths:

$$
\pi^{T}=(\underbrace{a^{*}, \ldots, a^{*}}_{T}, x^{*}), \quad \text { for all } T \geq 0,
$$

where $x_{i}^{*}=m_{i}^{j}$ for even $T$ and $x_{i}^{*}=m_{i}^{i}$ for odd $T$. In fact, the part of $\varphi^{j}(\cdot)$ we need is fully characterized by $v_{i}\left(\pi^{T}\right)$ for all even $T$ due to the following lemma:

Lemma 10 For any even $T \geq 0$, we have

$$
\begin{aligned}
& v_{i}\left(\pi^{T}\right)<v_{i}\left(\pi^{T+1}\right) \leq v_{i}\left(\pi^{T+2}\right)<d_{i}\left(a^{*}\right), \\
& v_{j}\left(\pi^{T}\right)>v_{j}\left(\pi^{T+2}\right)<v_{j}\left(\pi^{T+1}\right)<d_{j}\left(a^{*}\right) .
\end{aligned}
$$




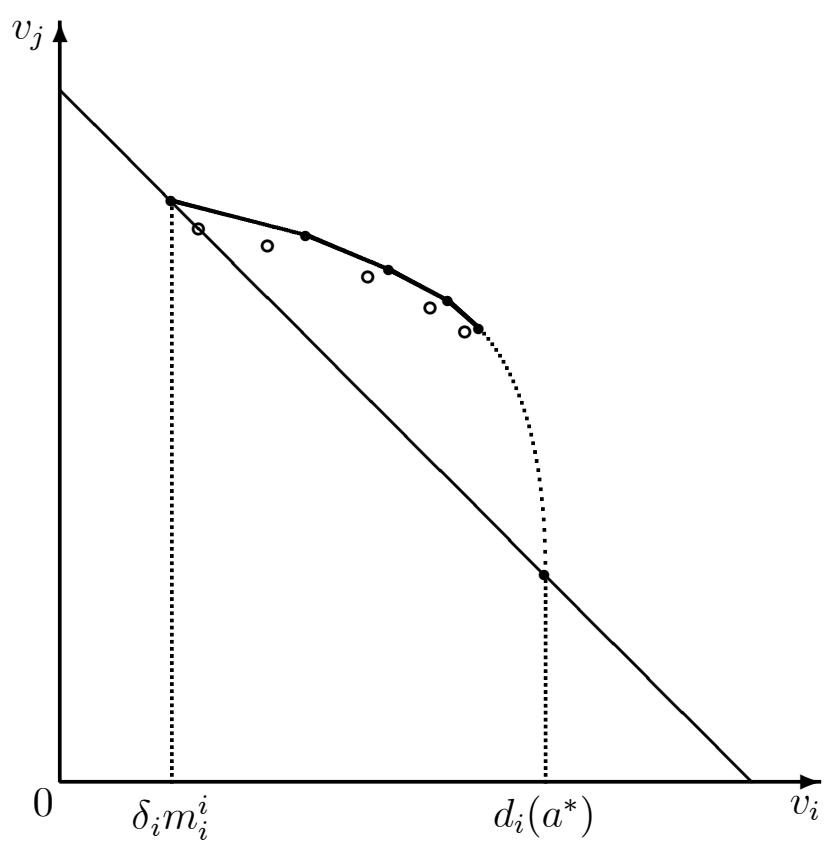

Figure 3: The payoff vectors $v\left(\pi^{T}\right)$ for $T \leq 8$.

The proof of Lemma 10 is given in the Appendix. (27) and (28) can be best illustrated by Figure 3, where $v\left(\pi^{T}\right)$ is represented by solid dots for even $T \leq 8$ and open dots for odd $T<8$. It implies that for any even $T \geq 0, v\left(\pi^{T+1}\right)$ is dominated by some convex combinations of $v\left(\pi^{T}\right)$ and $v\left(\pi^{T+2}\right)$. Intuitively, if the continuation path were associated with an odd $T$, then player $i$ would make a proposal along such a continuation, from which player $i$ could exploit his first-mover advantage. Consequently, such a continuation can never be effective in solving (4). For all even $T$, any convex combination of $v\left(\pi^{T}\right)$ and $v\left(\pi^{T+2}\right)$ can be achieved by a publicly correlated strategy between $\pi^{T}$ and $\pi^{T+2}$. For sufficiently large $\left(\delta_{i}, \delta_{j}\right) \in(0,1)^{2}$ and $\delta_{i}<\delta_{j}$, we have the following form of $\varphi^{j}\left(v_{i}\right)$ for $v_{i}$ sufficiently close to $\delta_{i} m_{i}^{i}$ :

$$
\varphi^{j}\left(v_{i}\right)=\min _{T \in 2 \mathbb{N}}\left\{v_{j}\left(\pi^{T}\right)+\frac{v_{j}\left(\pi^{T+2}\right)-v_{j}\left(\pi^{T}\right)}{v_{i}\left(\pi^{T+2}\right)-v_{i}\left(\pi^{T}\right)}\left[v_{i}-v_{i}\left(\pi^{T}\right)\right]\right\} .
$$

For every even $T \geq 0$, each function under the minimum in (29) can be written as

$$
1+\frac{\delta_{j}^{T}\left(1-\delta_{j}^{2}\right)\left(1-\delta_{i}^{T}\right)-\delta_{i}^{T}\left(1-\delta_{i}^{2}\right)\left(1-\delta_{j}^{T}\right)}{\delta_{i}^{T}\left(1-\delta_{i}^{2}\right)} d_{i}\left(a^{*}\right)-\frac{\delta_{j}^{T}\left(\delta_{j}^{2}-\delta_{i}^{2}\right)}{\left(1-\delta_{i}^{2}\right)} m_{i}^{i}-\frac{\delta_{j}^{T}\left(1-\delta_{j}^{2}\right)}{\delta_{i}^{T}\left(1-\delta_{i}^{2}\right)} v_{i} .
$$


This last formula is a linear and decreasing function of $m_{i}^{i}$ and $v_{i}$. Substituting (29) into (14), one can solve $m_{i}^{i}$ for the case of $\delta_{i}<\delta_{j}$.

We now present an example to demonstrate how to solve $m_{i}^{i}$. Consider a negotiation problem with the following $2 \times 2$ disagreement game for $\varepsilon \geq 0$ :

\begin{tabular}{|c|c|c|}
\hline Player 1 \Player 2 & $L$ & $R$ \\
\hline$U$ & $0.5,0.5$ & $-\varepsilon, 0.5$ \\
\hline$D$ & $0.5,0$ & $0,-1$ \\
\hline
\end{tabular}

where $a^{*}=(U, L)$. For simplicity, we consider pure actions only.

To support the SPE of Proposition 8 for $i=1$, two players would play $(U, L)$ in any odd period and $(D, R)$ in any even period. When $\delta_{1} \geq \delta_{2}$, both players behave as if in the alternating offer model with disagreement point $(0,0.5)$, from which player 1 receives $\frac{1-\delta_{2}}{1-\delta_{1} \delta_{2}} \cdot 0.5$ in any odd period. By the one-stage deviation principle, see e.g., Fudenberg and Tirole (1991), player 1 prefers to make such a proposal if and only if

$$
\frac{1-\delta_{2}}{1-\delta_{1} \delta_{2}} \cdot 0.5 \geq\left(1-\delta_{1}\right) \cdot 0.5+\delta_{1} \cdot \frac{1-\delta_{2}}{1-\delta_{1} \delta_{2}} \cdot 0.5 \quad \Leftrightarrow \quad \delta_{1} \geq \delta_{2} .
$$

For $\delta_{1}<\delta_{2}$, the SPE of Proposition 8 requires that only player 2 makes the least acceptable proposal to player 1 . Consequently, player 1 receives $\frac{1}{1+\delta_{1}} \cdot 0.5$ in any odd period, which is equal to player 1's present value from the infinite sequence of alternating disagreement outcomes. This SPE has been misidentified as player $i$ 's worst SPE when $\delta_{1}<\delta_{2}$. In what follows, we modify this SPE by replacing $(U, L)$ by $(U, R)$ in every odd period after player 1's rejection. Clearly, player 1 gains $\varepsilon$ in the disagreement game by deviating from $U$ to $D$. As we have argued, player 1 can be induced to play $U$ by a continuation SPE that is $\frac{1-\delta_{i}}{\delta_{i}} \varepsilon$ better than his worst SPE in the following period. We now derive player 1's worst SPE for the case of $\delta_{1}<\delta_{2}$ where the continuation involves $T=2$ periods delay in reaching an agreement. Consider the following strategy profile:

- In an odd period, player 1 demands

$$
x_{1}^{*}=\frac{1-\delta_{2}}{1-\delta_{1} \delta_{2}}\left[\frac{1}{2}+\frac{\delta_{2}}{\delta_{1}} \frac{1+\delta_{2}}{1+\delta_{1}} \varepsilon\right]
$$


and player 2 will reject if and only if player 1 demands more than $x_{1}^{*}$.

If player 1 demands more than $x_{1}^{*}$ and player 2 rejects, then $(U, R)$ will be played.

- In an even period, if player 1 deviates from $U$ in the last (odd) period, player 2 will offer $\delta_{1} x_{1}^{*}$ and player 1 will accept.

- Otherwise, with probability $1-p$, player 2 will offer $\delta_{1} x_{1}^{*}$ in the current even period, and with probability $p,(U, L)$ will be played for two periods, followed by player 2's offer $\delta_{1} x_{1}^{*}$. Player 1 accepts in both cases. In this equilibrium,

$$
p=\frac{1}{\delta_{1}\left(1-\delta_{1}\right)} \cdot \frac{\varepsilon}{0.5-\delta_{1} x_{1}^{*}}
$$

- In an even period, if player 1 rejects $\delta_{1} x_{1}^{*}$ (that should be accepted), then $(D, R)$ will be played once followed by player 1's demand $x_{1}^{*}$.

- If player 2 deviates from the strategies described above, then continuation will switch immediately to the stationary SPE of Proposition 1 from which player 1 receives 0.5.

To verify that the above strategy profile constitutes a SPE, first note that player 1 has no incentive to deviate from $(U, R)$ if his payoff from deviation is the same as what player 1 receives if he does not:

$$
\delta_{1}^{2} x_{1}^{*}=\left(1-\delta_{1}\right) \cdot(-\varepsilon)+\delta_{1}\left[(1-p) \delta_{1} x_{1}^{*}+p\left(0.5\left(1-\delta_{1}^{2}\right)+\delta_{1}^{3} x_{1}^{*}\right)\right]
$$

One can show that (33) holds for $p$ as given by (32). Next, player 1 should demand $x_{1}^{*}$ rather than making an unacceptable proposal,

$$
x_{1}^{*} \geq\left(1-\delta_{1}\right) \cdot(-\varepsilon)+\delta_{1}\left[(1-p) \delta_{1} x_{1}^{*}+p\left(0.5\left(1-\delta_{1}^{2}\right)+\delta_{1}^{3} x_{1}^{*}\right)\right]=\delta_{1}^{2} x_{1}^{*},
$$

which follows from (33). Lastly, player 1 cannot demand more than $x_{1}^{*}$ since $1-x_{1}^{*}$ is exactly equal to player 2's continuation payoff after rejecting any demand higher than $x_{1}^{*}$ :

$$
1-x_{1}^{*}=0.5\left(1-\delta_{2}\right)+\delta_{2}\left[(1-p)\left(1-\delta_{1} x_{1}^{*}\right)+p\left[0.5\left(1-\delta_{2}^{2}\right)+\delta_{2}^{2}\left(1-\delta_{1} x_{1}^{*}\right)\right]\right] .
$$




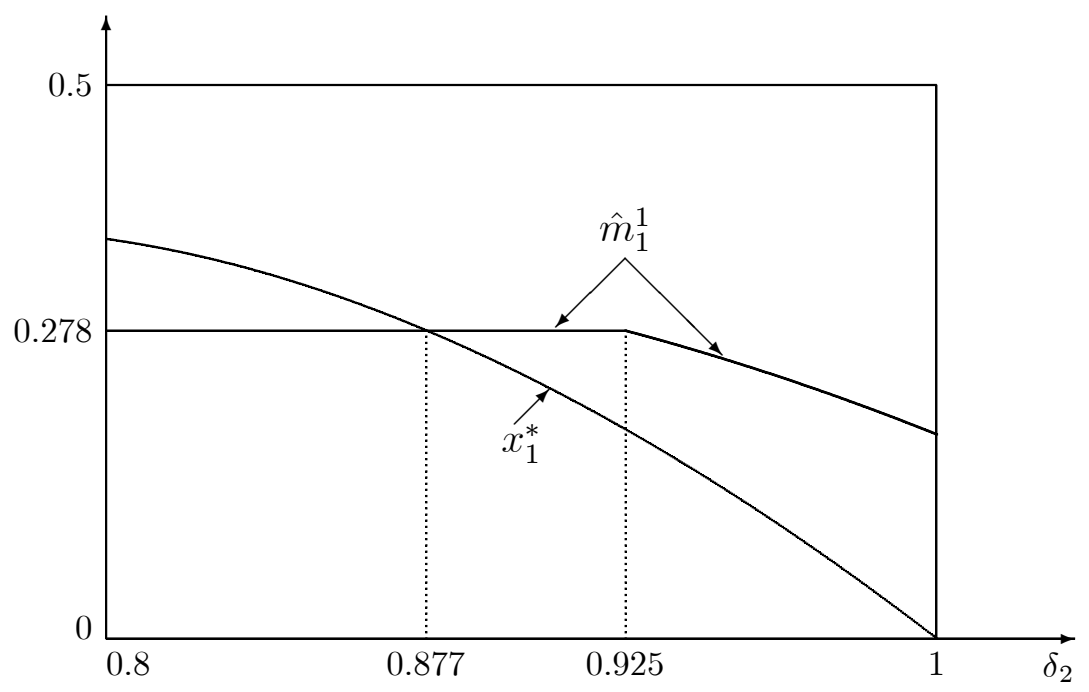

Figure 4: Plot of $x_{1}^{*}$ with respect to $\delta_{2} \in\left(\delta_{1}, 1\right)$ for $\delta_{1}=0.8$ and $\varepsilon=0.15$.

In fact, (33) and (34) yield $x_{1}^{*}$ and $p$ as given by (31) and (32), respectively.

For $\delta_{1}=0.8$ and $\varepsilon=0.15$, Figure 4 shows $x_{1}^{*}$ is less than $\frac{d_{1}\left(a^{*}\right)}{1+\delta}=0.278$ for all $\delta_{2} \in$ $(0.877,1)$. When the difference between the players' time preferences is not significant enough such as $\delta_{2} \in(0.8,0.877)$, it would not be optimal to have delay in the continuation while compensating player 1. In such a case, the non-stationary SPE of Proposition 8 is likely to be player 1's worst SPE. However, such incidence diminishes as the value of $\varepsilon$ decreases. Figure 4 also illustrates

$$
\hat{m}_{1}^{1}=\min \left\{\frac{1}{1+\delta_{1}} \frac{1}{2}, \frac{1-\delta_{2}}{1-\delta_{1} \delta_{2}}\left[\frac{1}{2}+\frac{\delta_{2}}{\delta_{1}} \frac{1-\delta_{1}}{1-\delta_{2}} \varepsilon\right]\right\}
$$

as defined by (22). In this example, $(U, R)$ supports $\hat{m}_{1}^{1}$ for $\delta_{1}=0.8$ and $\delta_{2} \geq 0.925$. Figure 4 shows that $m_{1}^{1}<\hat{m}_{1}^{1}$ for $\delta_{1}=0.8$ and $\delta_{2}>0.877$.

To conclude, the results obtained in this section will not be qualitatively affected if $d\left(a^{*}\right)$ is below the bargaining frontier since $\varphi^{j}(\cdot)$ by $(29)$ will remain the same. If $d\left(a^{*}\right)$ is strictly below the bargaining frontier, however, it will enlarge the set of discount factors for which delay does not occur in supporting the less patient players' worst SPE. For those discount factors, Proposition 9 prevails. At the extreme when $d\left(a^{*}\right)=0$, the negotiation model is 
equivalent to the alternating-offer model in Rubinstein (1982), and all continuation payoffs are bounded by the bargaining frontier for all discount factors.

\section{Concluding Remarks}

In this paper, we pin down what has been overlooked in previous studies: Players may trade their difference in time preferences to become better off than what is attainable from immediate agreements. Such a trade is possible only if they disagree for some periods. Therefore, disagreement is not necessarily inefficient. We show that this matters in a player's worst equilibrium and we incorporate this line of argument in our analysis.

In this paper, we did not allow for nonstationary contracts due to a number of considerations. First, allowing for nonstationary contracts would make it impossible to tie our results to the existing literature where only stationary contracts are permitted. Second, in this model it is unclear what to allow if the restriction on stationary contracts is relaxed. When the unit simplex is the set of all permissible per-period contracts, the bargaining frontier resulting from nonstationary contracts merely consists of the most extreme per-period contracts, namely $(0,1)$ and $(1,0)$. When only stationary contracts are allowed, it is without loss of any generality to restrict contracts on the unit simplex since all the other contracts are not individually rational with respect to the minimax value in the disagreement game (normalized to 0 ). If the restriction on stationary contracts were relaxed, there would be no reason why players should negotiate only nonstationary contracts resulted from the unit simplex. In other words, there is no obvious and sensible bounded set of per-period contracts that is consistent with the notion of unrestricted nonstationary contracts. The lack of such a bounded set of per-period contracts implies that there is no well-defined and finite bargaining frontier, i.e., the Pareto frontier of all possible contracts. Third, even if there is a bounded set of per-period contracts that nonstationary contracts can be based on, such as the unit simplex in wage negotiations, any Pareto efficient contract requires that the firm receive nothing after some finite periods when the firm is less patient than the union. Such contracts may not be practical or legal in reality. Therefore, real-life negotiation problems 
may impose a more restricted contract space than what is mathematically needed. Fourth, for any bounded set of nonstationary contracts, there are always disagreement games such that the phenomena we study in this paper occur.

In the negotiation model, restrictions on the contract space matter in the equilibrium analysis, because they prevent players from trading their difference in time preferences. Therefore, the bargaining frontier associated with any restricted contract space can be inefficient. Our analysis is robust to bargaining frontiers other than the unit simplex and must be applied whenever some trade of the difference in time preferences makes the players better off than from reaching an immediate agreement. Therefore, one should not put too much emphasis on the numerical values we get, but what is rather important is how players behave in those extreme situations.

\section{Appendix: Proofs}

Proof of Proposition 7: The proof is constructive. Without loss of generality, assume that when $\delta_{i} \geq \delta^{\prime}$ and $\delta_{j} \geq \delta^{\prime}$ for some $\delta^{\prime} \in(0,1)$, there exists a SPE from which player $j$ 's payoff is less than $1-\hat{m}_{i}^{i}-\varepsilon$ for some $\varepsilon>0$, i.e., $m_{j}^{j} \leq 1-\hat{m}_{i}^{i}-\varepsilon$. Let $m_{i}^{i}$ be the least fixed point of $\Lambda(\cdot)$, which generally depends on $\left(\delta_{i}, \delta_{j}\right) \in(0,1)^{2}$. For sufficiently large $\delta^{\prime \prime}$ and $\delta_{j}>\delta_{i} \geq \delta^{\prime \prime}$,

we have $\varphi^{j}\left(v_{i}\right)$ depends only on $m_{i}^{i}$ for all $v_{i} \in\left[\delta_{i} m_{i}^{i}, \delta_{i} m_{i}^{i}+\frac{1-\delta_{i}}{\delta_{i}} \max _{a \in A}\left[g_{i}(a)-d_{i}(a)\right]\right]$ and

$$
\varphi^{j}\left(\delta_{i} m_{i}^{i}+\frac{1-\delta_{i}}{\delta_{i}} \max _{a \in A}\left[g_{i}(a)-d_{i}(a)\right]\right) \geq m_{j}^{j}+\frac{\varepsilon}{2} .
$$

From the definition of $\varphi^{j}(\cdot),\left(v_{i}, \varphi^{j}\left(v_{i}\right)\right)$ is a SPE payoff vector for all

$$
v_{i} \in\left[\delta_{i} m_{i}^{i}, \delta_{i} m_{i}^{i}+\frac{1-\delta_{i}}{\delta_{i}} \max _{a \in A}\left[g_{i}(a)-d_{i}(a)\right]\right]
$$

as long as $\delta_{j}>\delta_{i} \geq \max \left\{\delta^{\prime}, \delta^{\prime \prime}\right\}$ and $m_{i}^{i}$ can be supported as player $i$ 's SPE payoff. Choose $\hat{\delta} \geq \max \left\{\delta^{\prime}, \delta^{\prime \prime}\right\}$ sufficiently large so that $\frac{1-\hat{\delta}}{\hat{\delta}} \max _{a \in A}\left[g_{j}(a)-d_{j}(a)\right] \leq \frac{\varepsilon}{2}$. In other words, if $\delta_{j} \geq \hat{\delta}$ then player $j$ will not deviate in the disagreement game as long as player $j$ 's average loss in the continuation payoffs is no less than $\frac{\varepsilon}{2}$. 
For all $\delta_{j}>\delta_{i} \geq \hat{\delta}$, let $m_{i}^{i}$ be the least fixed point of $\Lambda(\cdot)$ and $\hat{a} \in A$ be the corresponding solution to (21). We have the following two cases to examine:

Case 1: $m_{i}^{i}=\left(1-\delta_{i}\right) g_{i}(\hat{a})+\delta_{i}^{2} m_{i}^{i}$.

Consider the following strategy profile: Player $i$ makes an unacceptable offer (such as demands $m_{i}^{i}$ or more). Player $j$ rejects if and only if player $i$ offers less than

$$
\left(1-\delta_{j}\right) d_{j}(\hat{a})+\delta_{j} \varphi^{j}\left(\delta_{i} m_{i}^{i}+\frac{1-\delta_{i}}{\delta_{i}}\left[g_{i}(\hat{a})-d_{i}(\hat{a})\right]\right) \geq 1-m_{i}^{i},
$$

followed by $\hat{a}$ once. If player $i$ deviates from $\hat{a}$, player $j$ will offer $\delta_{i} m_{i}^{i}$ and player $i$ will accept in the following period. Otherwise, the continuation SPE in the following period will be on the Pareto frontier of $E^{j}$ from which player $i$ will receive $\delta_{i} m_{i}^{i}+\frac{1-\delta_{i}}{\delta_{i}}\left[g_{i}(\hat{a})-d_{i}(\hat{a})\right]$. If player $j$ deviates from what is described above, player $j$ will be punished by the SPE provided at the beginning of this proof from which his payoff will not be higher than $1-\hat{m}_{i}^{i}-\varepsilon$.

We now verify sequential rationality. It is clear from the construction that no one deviates in the proposing and responding stages. For example, player $i$ has to offer at least

$$
\left(1-\delta_{j}\right) d_{j}(\hat{a})+\delta_{j} \varphi^{j}\left(\delta_{i} m_{i}^{i}+\frac{1-\delta_{i}}{\delta_{i}}\left[g_{i}(\hat{a})-d_{i}(\hat{a})\right]\right)
$$

in order to induce player $j$ to accept, from which player $i$ receives less than $m_{i}^{i}$. Player $i$ will not deviate from $\hat{a}$ because

$$
\left(1-\delta_{i}\right) g_{i}(\hat{a})+\delta_{i}\left(\delta_{i} m_{i}^{i}\right)=\left(1-\delta_{i}\right) d_{i}(\hat{a})+\delta_{i}\left(\delta_{i} m_{i}^{i}+\frac{1-\delta_{i}}{\delta_{i}}\left[g_{i}(\hat{a})-d_{i}(\hat{a})\right]\right) .
$$

Case 2: $m_{i}^{i}=1-\left(1-\delta_{j}\right) d_{j}(\hat{a})-\delta_{j} \varphi^{j}\left(\delta_{i} m_{i}^{i}+\frac{1-\delta_{i}}{\delta_{i}}\left[g_{i}(\hat{a})-d_{i}(\hat{a})\right]\right)$.

Consider the following strategy profile: Player $i$ demands $m_{i}^{i}$. Player $j$ rejects if and only if player $i$ demands more than $m_{i}^{i}$. If player $i$ demands more and player $j$ rejects (which should not occur), two players will play $\hat{a}$, and the continuations will the same as those in Case 1 for the corresponding histories.

Similar to Case 1, no one will deviate after player $i$ demands more $m_{i}^{i}$ and player $j$ rejects. If player $i$ demands more than $m_{i}^{i}$ at the beginning, player $j$ will reject, and player $i$ will receive

$$
\left(1-\delta_{i}\right) d_{i}(\hat{a})+\delta_{i}\left[\delta_{i} m_{i}^{i}+\frac{1-\delta_{i}}{\delta_{i}}\left[g_{i}(\hat{a})-d_{i}(\hat{a})\right]\right] \leq m_{i}^{i} .
$$


Therefore, player $i$ will demand $m_{i}^{i}$, which will be accepted by player $j$. In summary, no one has incentive to deviate when player $i$ is supposed to demand $m_{i}^{i}$.

We have shown that in either case, there is an equilibrium where player $i$ receives $m_{i}^{i}$, the least fixed point of $\Lambda(\cdot)$, when making a proposal.

Proof of Lemma 10: For any even $T \geq 0$, we have $v_{i}\left(\pi^{T}\right)<v_{i}\left(\pi^{T+1}\right) \leq v_{i}\left(\pi^{T+2}\right)$;

$$
\begin{aligned}
\left(1-\delta_{i}^{T}\right) d_{i}\left(a^{*}\right)+\delta_{i}^{T+1} m_{i}^{i} & <\left(1-\delta_{i}^{T+1}\right) d_{i}\left(a^{*}\right)+\delta_{i}^{T+1} m_{i}^{i}, \\
\left(1-\delta_{i}^{T+1}\right) d_{i}\left(a^{*}\right)+\delta_{i}^{T+1} m_{i}^{i} & \leq\left(1-\delta_{i}^{T+2}\right) d_{i}\left(a^{*}\right)+\delta_{i}^{T+3} m_{i}^{i},
\end{aligned}
$$

where the first inequality is trivial, and the second inequality is due to $m_{i}^{i} \leq \frac{1}{1+\delta_{i}} d_{i}\left(a^{*}\right)$ by Proposition 8. Comparing player $j$ 's payoffs, we have $v_{j}\left(\pi^{T}\right)>v_{j}\left(\pi^{T+2}\right) \geq v_{i}\left(\pi^{T+1}\right)$;

$$
\begin{aligned}
\left(1-\delta_{j}^{T}\right) d_{j}\left(a^{*}\right)+\delta_{j}^{T}\left(1-\delta_{i} m_{i}^{i}\right) & >\left(1-\delta_{j}^{T+2}\right) d_{j}\left(a^{*}\right)+\delta_{j}^{T+2}\left(1-\delta_{i} m_{i}^{i}\right), \\
\left(1-\delta_{j}^{T+2}\right) d_{j}\left(a^{*}\right)+\delta_{j}^{T+2}\left(1-\delta_{i} m_{i}^{i}\right) & \geq\left(1-\delta_{j}^{T+1}\right) d_{j}\left(a^{*}\right)+\delta_{j}^{T+1}\left(1-m_{i}^{i}\right),
\end{aligned}
$$

where the first inequality is trivial, and second inequality is due to $\frac{1-\delta_{j}}{1-\delta_{i} \delta_{j}}\left[1-d_{j}\left(a^{*}\right)\right] \leq m_{i}^{i}$ by Proposition 9.

\section{References}

Abreu, D., D. Pearce, and E. Stacchetti (1986). Optimal cartel equilibria with imperfect monitoring. Journal of Economic Theory 39, 251-269.

Abreu, D., D. Pearce, and E. Stacchetti (1990). Toward a theory of discounted repeated games with imperfect monitoring. Econometrica 58, 1041-1063.

Bewley, T. (1972). Existence of equilibria in economies with infinitely many commodities. Journal of Economic Theory 4, 514-540.

Binmore, K. (1987). Perfect equilibria in bargaining models. In K. Binmore and P. Dasgupta (Eds.), The economics of bargaining, pp. 77-105. Oxford: Basil Blackwell.

Bolt, W. (1993). Wage bargaining in the Fernandez and Glazer model: A comment. Tinbergen Institute Discussion Paper No. 93-240, Tinbergen Institute. 
Bolt, W. (1995). Striking for a bargain between two completely informed agents: Comment. American Economic Review 85, 1344-1347.

Busch, L.-A. and Q. Wen (1995). Perfect equilibria in a negotiation model. Econometrica $63,545-565$.

Busch, L.-A. and Q. Wen (2001). Negotiations games with unobservable mixed disagreement actions. Journal of Mathematical Economics 35, 563-579.

Farrel, J. and G. Saloner (1985). Standardization, compatibility, and innovation. RAND Journal of Economics 16, 70-83.

Fernandez, R. and J. Glazer (1991). Striking for a bargain between two completely informed agents. American Economic Review 81, 240-252.

Fudenberg, D. and J. Tirole (1991). Game theory. London: MIT Press.

Haller, H. (1991). Wage bargaining as a strategic game. In R. Selten (Ed.), Game theoretic equilibrium models III: Strategic bargaining, pp. 230-241. Berlin: Springer Verlag.

Haller, H. and S. Holden (1990). A letter to the editor on wage bargaining. Journal of Economic Theory 52, 232-236.

Houba, H. (1997). The policy bargaining model. Journal of Mathematical Economics 28, $1-27$.

Houba, H. and W. Bolt (2000). Holdouts, backdating and wage negotiations. European Economic Review 44, 1783-1800.

Houba, H. and W. Bolt (2002). Credible threats in negotiations; A game theoretic approach. Theory and Decision Library. Series C: Game Theory Mathematical Programming and Operations Research, vol. 32. Norwell, Mass. and Dordrecht: Kluwer Academic.

Houba, H. and Q. Wen (2006). Different time preferences and non-stationary contracts in negotiations. Economics Letters 91, 273-279.

Lehrer, E. and A. Pauzner (1999). Repeated games with different time preferences. Econometrica $67,393-412$. 
Mailath, G. and L. Samuelson (2006). Repeated Games and Reputations: Long-Run Relationships. Oxford: Oxford University Press.

Muthoo, A. (1999). Bargaining theory with applications. Cambridge: Cambridge University Press.

Nash, J. (1953). Two-person cooperative games. Econometrica 21, 128-140.

van Damme, E. (1991). Stability and perfection of Nash equilibria (Second ed.). Berlin: Springer Verlag.

Ramsey, F. (1928). A mathematical theory of saving. Economic Journal 38, 453-459.

Rubinstein, A. (1982). Perfect equilibrium in a bargaining model. Econometrica 50, 97109.

Shaked, A. and J. Sutton (1984). Involuntary unemployment as a perfect equilibrium in a bargaining model. Econometrica 62, 1351-1364.

Slantchev, B. (2003). The power to hurt: costly conflict with completely informed states. American Political Science Review 97, 123-133.

Takahashi, S. (2005). Infinite horizon common interest games with perfect information. Games and Economic Behavior 53, 231-247. 